\title{
Utility of Inflammatory Marker- and Nutritional Status-based Prognostic Factors for Predicting the Prognosis of Stage IV Gastric Cancer Patients Undergoing Non-curative Surgery
}

\author{
KENJI MIMATSU ${ }^{1}$, NOBUTADA FUKINO ${ }^{1}$, YASUO OGASAWARA ${ }^{1}$, YOKO SAINO ${ }^{2}$ and TAKATSUGU OIDA ${ }^{3}$ \\ Departments of ${ }^{1}$ Surgery, and ${ }^{2}$ Nutrition, \\ Japan Community Health Care Organization Yokohama Central Hospital, Kanagawa, Japan; \\ ${ }^{3}$ Department of Surgery, Kiba Hospital, Tokyo, Japan
}

\begin{abstract}
Background/Aim: The present study aimed to compare the utility of various inflammatory marker-and nutritional status-based prognostic factors, including many previous established prognostic factors, for predicting the prognosis of stage IV gastric cancer patients undergoing noncurative surgery. Patients and Methods: A total of 33 patients with stage IV gastric cancer who had undergone palliative gastrectomy and gastrojejunostomy were included in the study. Univariate and multivariate analyses were performed to evaluate the relationships between the mGPS, PNI, NLR, $P L R$, the CONUT, various clinicopathological factors and cancer-specific survival (CS). Results: Among patients who received non-curative surgery, univariate analysis of $C S$ identified the following significant risk factors: chemotherapy, $m G P S$ and NLR, and multivariate analysis revealed that the mGPS was independently associated with CS. Conclusion: The $m G P S$ was a more useful prognostic factor than the PNI, NLR, PLR and CONUT in patients undergoing non-curative surgery for stage IV gastric cancer.
\end{abstract}

The aim of palliative surgery for stage IV gastric cancer is to relieve distressing symptoms, including cancer pain, tumor hemorrhage and inability to eat (1). However, it is controversial whether non-curative surgical treatment improves the prognosis of patients with incurable stage IV gastric cancer. The prognosis of cancer patients is determined by many factors, among which tumor- and patient-related

Correspondence to: Kenji Mimatsu, Department of Surgery, Japan Community Health care Organization Yokohama Central Hospital, 268 Yamashita-cho Naka-ku Yokohama, Kanagawa, 231-8553, Japan. Tel: +81 456411921, Fax: +81 456719872, e-mail: mimatsukenji@yokohama.jcho.jp

Key Words: Stage IV gastric cancer, non-curative surgery, Glasgow prognostic score, neutrophil to lymphocyte ratio. factors are particularly important; their use as prognostic factors remains problematic due to the wide range of tumorand patient-related factors and because the results are subject to bias. Thus, in order to establish the optimal medical treatment and determine the optimal timing of surgery it is crucial to identify effective markers of the immunological and nutritional status that can be used to predict the prognosis. Furthermore, it is necessary to find factors that can accurately predict the response to surgical treatment in patients with stage IV gastric cancer in order to identify the patients who are more likely to benefit from non-curative surgery.

In the past few decades, investigators have demonstrated that the presence of a systemic inflammatory response and the preoperative immunonutritional status are associated with a poor prognosis in patients with various types of cancer (2-5). The Glasgow Prognostic Score (GPS) (5-12), the Prognostic Nutritional Index (PNI) (13-15), the neutrophil lymphocyte ratio (NLR) $(10,16-21)$, the platelet lymphocyte ratio (PLR) (21-24) and the controlling nutritional status (CONUT) (2527) have been reported to have prognostic value in patients with many types of cancer. Some studies have demonstrated that these inflammatory and nutritional factors may predict the prognosis of patients with advanced gastric cancer (10-12, 1722). However, there have been no reports on the factors that are most useful for predicting the prognosis of patients with stage IV gastric cancer after non-curative surgery, including gastrectomy and gastrojejunostomy. Thus, in the present study, we investigated the usefulness of markers of inflammation and the nutritional status for predicting the prognosis of patients undergoing non-curative surgery for incurable stage IV gastric cancer.

\section{Patients and Methods}

A total of 271 gastric cancer patients underwent surgical resection or gastrojejunostomy in our Institution between January 2006 and December 2016. Among these patients, 49 patients with stage IV gastric cancer were enrolled in the present study. This study was 
approved by the ethics committee of our hospital (IRB No. 201704). All of the medical records were reviewed retrospectively. Patients who died within 30 days of surgery and who had other malignancies were excluded from the study. We excluded 16 patients for the following reasons: death within 30 days $(n=2)$, concurrent malignancies $(n=3)$ and incomplete clinical data $(n=11)$. The remaining 33 cases included in this study had adequate clinical information and follow-up data. Non-curative surgery, including gastrectomy and gastrojejunostomy, was defined as described previously (12). The pathological diagnoses and classifications were made according to the 7th edition of the UICC TNM Classification of Malignant Tumors (28).

Blood samples were obtained within a week before surgery to measure the white blood cell, neutrophil and lymphocyte counts, and the C-reactive protein (CRP), albumin, total cholesterol, carcinoma embryonic antigen (CEA) and carbohydrate antigen (CA) 19-9 levels. Patients were evaluated using the following values: the modified GPS (mGPS) (patients with both an elevated CRP level [ $>0.5 \mathrm{mg} / \mathrm{dl}]$ and hypoalbuminemia $[<3.5 \mathrm{~g} / \mathrm{dl}]$ were assigned an mGPS of 2; patients with one of these blood chemistry abnormalities were assigned a score of 1 ; and those with no abnormalities were assigned a score of 0$)(29)$, the PNI (10xserum albumin level $[\mathrm{g} / \mathrm{dl}]+0.005 \times$ peripheral lymphocyte count $\left.\left[/ \mathrm{mm}^{3}\right]\right)$ (30), the NLR (neutrophils/lymphocytes $\left[\mathrm{mm}^{3} / \mathrm{mm}^{3}\right]$ ), the PLR (platelet/lymphocytes $\left[\mathrm{mm}^{3} / \mathrm{mm}^{3}\right]$ ) and the CONUT (a score composed of the serum albumin concentration, the total peripheral lymphocyte count, and the total cholesterol concentration) (31).

We established effective cutoff levels for the different indexes in which a large amount of difference was observed between two groups. For example, the mGPS was tested at $0-1$ and 2 , or 0 and 1-2; the PNI was tested at set cutoff levels of 40, 45, 50; the NLR was tested at set cutoff levels of 2.0, 2.5, 3.0, 3.5, 4.0; and the PLR was tested at set cutoff levels of $150,200,250$; the CONUT was tested at cutoff levels of $\leq 3 v s . \geq 4$, or $\leq 4 v s$. $\geq 5$, or $\leq 5 v s$. $\geq 6$. Then, we divided each group based on the preoperative mGPS (0-1 vs. 2), PNI ( $>40 v s . \leq 40)$, NLR (<2.5 vs. $\geq 2.5)$, PLR ( $\leq 200$ and $>200)$, CONUT ( $\leq 4 v s . \geq 5$ ).

The potential prognostic factors for advanced gastric cancer were as follows: age ( $<70$ years $v s . \geq 70$ years); sex (male $v s$. female); body mass index $(<22 v s . \geq 22)$; Eastern Cooperative Oncology Group performance status (0-1 vs. $\geq 2)$; American Society of Anesthesiologists (ASA) physical status (0-1 vs. $\geq 2$ ); histological subtype (well and moderately differentiated adenocarcinoma vs. poorly differentiated and undifferentiated adenocarcinoma); tumor depth ( $\leq \mathrm{T} 3 v s$. T4); number of metastatic sites ( $\leq 1 v s . \geq 2)$, metastatic sites (such as the liver, lung, and peritoneum); peritoneal cytology (positive $v s$. negative); CEA level ( $<5 v s . \geq 5$ ); CA19-9 level ( $\leq 37 v s .>37)$; mGPS (0-1 vs. 2$)$; PNI (>40 vs. $\leq 40)$; NLR $(<2.5 v s . \geq 2.5)$; PLR ( $\leq 200$ and $>200)$; CONUT ( $\leq 4 v s . \geq 5)$; surgical treatment (gastrectomy vs. gastrojejunostomy); chemotherapy (performed $v s$. not performed); and the presence or absence of postoperative complications (defined as Grade $>$ II according to the Clavien-Dindo classification (32)). The associations between the mGPS, PNI, NLR, PLR, CONUT, the clinicopathological parameters and overall survival (OS) were assessed.

Intergroup comparisons were performed using the Mann-Whitney $U$-test for continuous and ordinal variables, and the chi-squared test and Fisher's exact test for categorical variables. The OS rates were calculated using the Kaplan-Meier method, and the differences in survival rates were compared using the log-rank test. OS was

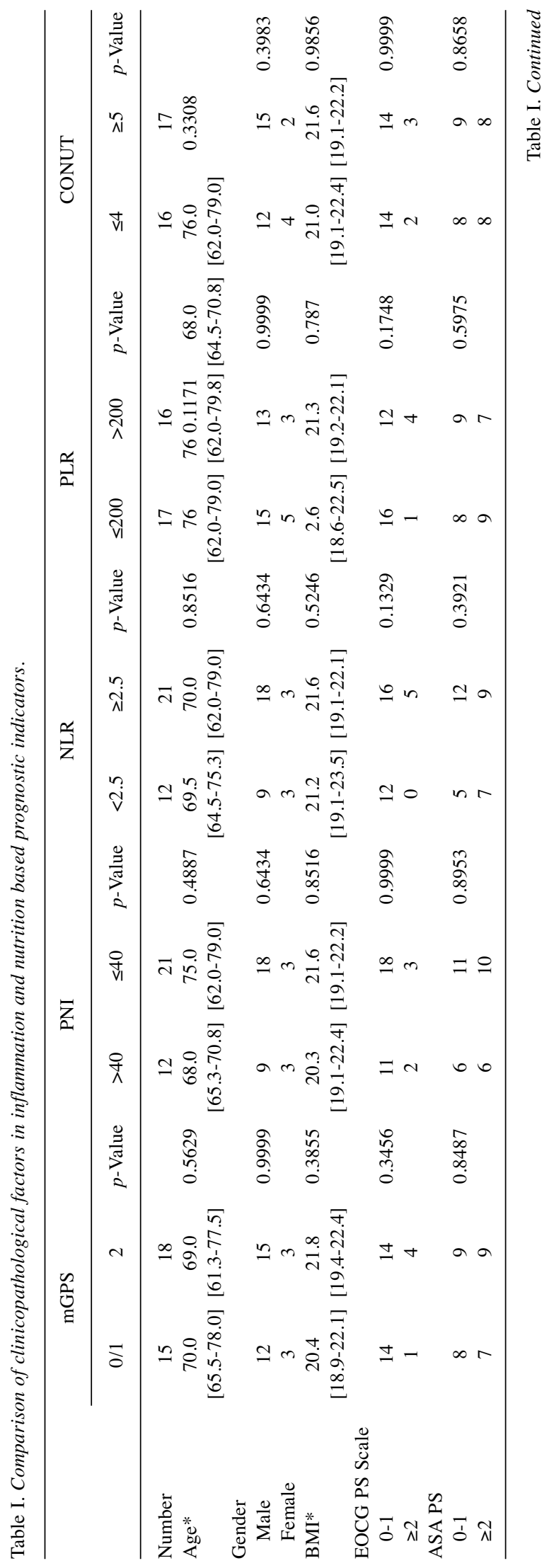




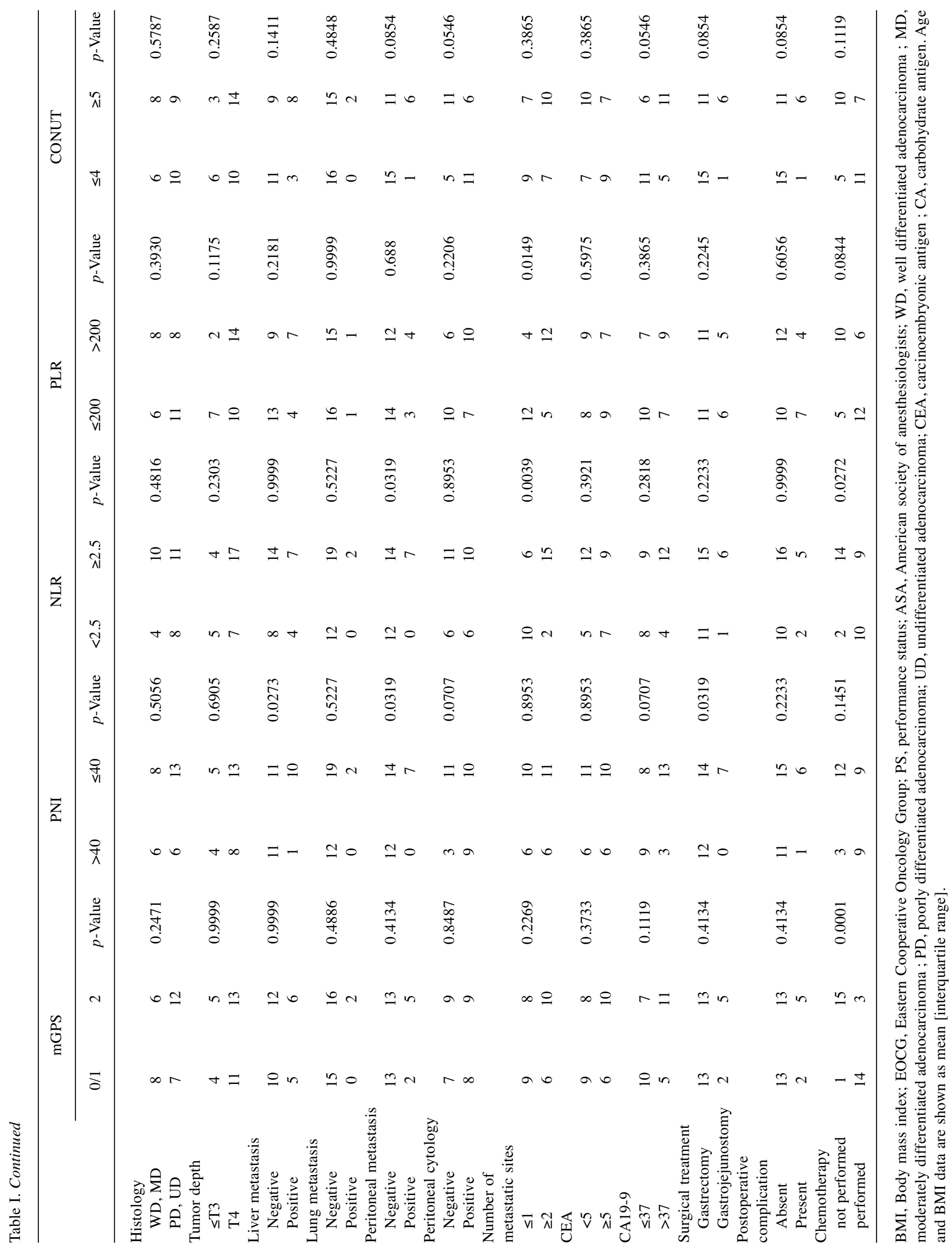


defined as the time from the first day of surgical treatment until death. Both univariate and multivariate analyses were performed using Cox's proportional hazards regression to assess the potential prognostic factors. $p$-Values of $<0.05$ were considered to indicate statistical significance in all of the analyses. All of the statistical analyses were performed using the IBM SPSS Statistics for Windows software program (Version 22.0, IBM Corporation, Armonk, NY, USA).

\section{Results}

Patient characteristics. Table I summarizes the background information of the patients with each prognostic factor. The rates of liver and peritoneal metastasis were significantly lower, whereas the incidence of gastrectomy in the PNI $>40$ group was significantly higher than that in the PNI $\leq 40$ group. All patients in the PNI $>40$ group underwent gastrectomy. The rate of peritoneal metastasis in the NLR $\geq 2.5$ group was significantly higher than that in the NLR $<2.5$ group and the number of metastatic sites was significantly increased in the NLR $\geq 2.5$ and PLR $>200$ groups. The incidence of chemotherapy in the mGPS (0-1) and NLR $<2.5$ groups was significantly higher than that in the mGPS (2) and NLR $\geq 2.5$ groups, respectively. There were no significant differences in the clinicopathological factors of the CONUT $\leq 4$ and CONUT $\geq 5$ groups.

The comparison of the survival rates. Thirty-one patients died and two were censored at the last date of follow-up. The median OS of all cases was 193 days. The OS rates of the mGPS $(0-1)(p=0.0001)$, PNI $>40(p=0.047)$, and NLR $<2.5$ $(p=0.013)$ groups were significantly better than those of their counterparts (Figure 1a, b and c).

Analysis of prognostic factors for OS. To obtain the adjusted hazards ratio for survival, a multivariate Cox proportional hazards model was fitted to the data after the backward elimination of candidate variables. Table II shows the relationships between the clinicopathological factors and OS among patients with incurable stage IV gastric cancer who underwent non-curative surgical treatment. The univariate analyses revealed significant differences in chemotherapy $(p=0.001)$, mGPS $(p=0.0001)$ and NLR $(p=0.012)$ while a multivariate analysis of the clinicopathological factors that showed significant differences in the univariate analyses revealed that mGPS $(\mathrm{HR}=0.371,95 \% \mathrm{CI}=0.181-0.76$, $p=0.007)$ was the only independent prognostic factor that was significantly associated with OS (Table II).

\section{Discussion}

Serum albumin concentration is well known to be a reliable indicator of the nutritional status and state of systemic inflammation (33). A low serum albumin level is considered to be associated with various cancer survival outcomes. On the other hand, serum CRP elevation, which indicates the presence of systemic inflammation, has been shown to be an independent prognostic factor in gastric cancer (34). The GPS combines these two variables (the serum albumin level and the CRP level). Thus, the GPS is considered useful as it reflects the degree of malnutrition and inflammation, which are influenced by the development of cancer. Several studies have reported that the GPS is a useful prognostic factor in gastric cancer (8-12). Nozoe et al. (8) reported that a high GPS was independently associated with a worse prognosis in gastric cancer patients undergoing curative resection. Kubota et al. (9) reported that the GPS was a significant predictor of short- and long-term survival in patients with stage I-III gastric cancer who were undergoing curative surgery. We also previously reported that the MGPS was a significant prognostic marker in patients undergoing palliative surgery for stage IV gastric cancer (12).

Wang et al. (11) revealed that the GPS was superior to the NLR and PLR and that it was associated with OS in stage III gastric cancer patients undergoing potentially curative resection. Liu et al. (6) compared the prognostic value of different nutrition- and inflammation-based markers using an ROC analysis. They revealed that the C-reactive protein/ albumin ratio was the best predictor of the survival of gastric cancer patients after curative resection. These results suggested that the inflammatory marker, which consisted of serum albumin and CRP, is better prognostic factor than the other inflammation and nutritional makers in patients with gastric cancer undergoing curative resection. However, there have been no reports of factors that predicted better survival in stage IV gastric cancer patients undergoing non-curative surgery. The results of the present study suggest that the mGPS may be a more useful prognostic factor than the PNI, NLR, PLR and CONUT for such patients.

The PNI was initially designed by Buzby et al. (35) to assess the immunological and nutritional aspects of patients who underwent surgical treatment for diseases of the digestive tract. Onodera et al. (30) proposed the modified PNI, which was calculated using the two values: the serum albumin concentration and the lymphocyte count in the peripheral blood. Recently, the PNI has been widely used to assess the preoperative condition and predict the surgical risk (including anastomotic deficiency) in patients undergoing surgery for gastrointestinal malignancies. Migita et al. (13) revealed that the overall and relapse-free survival rates in a PNI-low group were significantly lower than those in a PNIhigh group among patients with stage I and stage III gastric cancer. A meta-analysis revealed that the PNI was significantly associated with poor OS in patients with stage I, II and III gastric cancer, but not in patients with stage IV gastric cancer (14). These results suggest that a low preoperative PNI has little impact on survival in patients with 
a
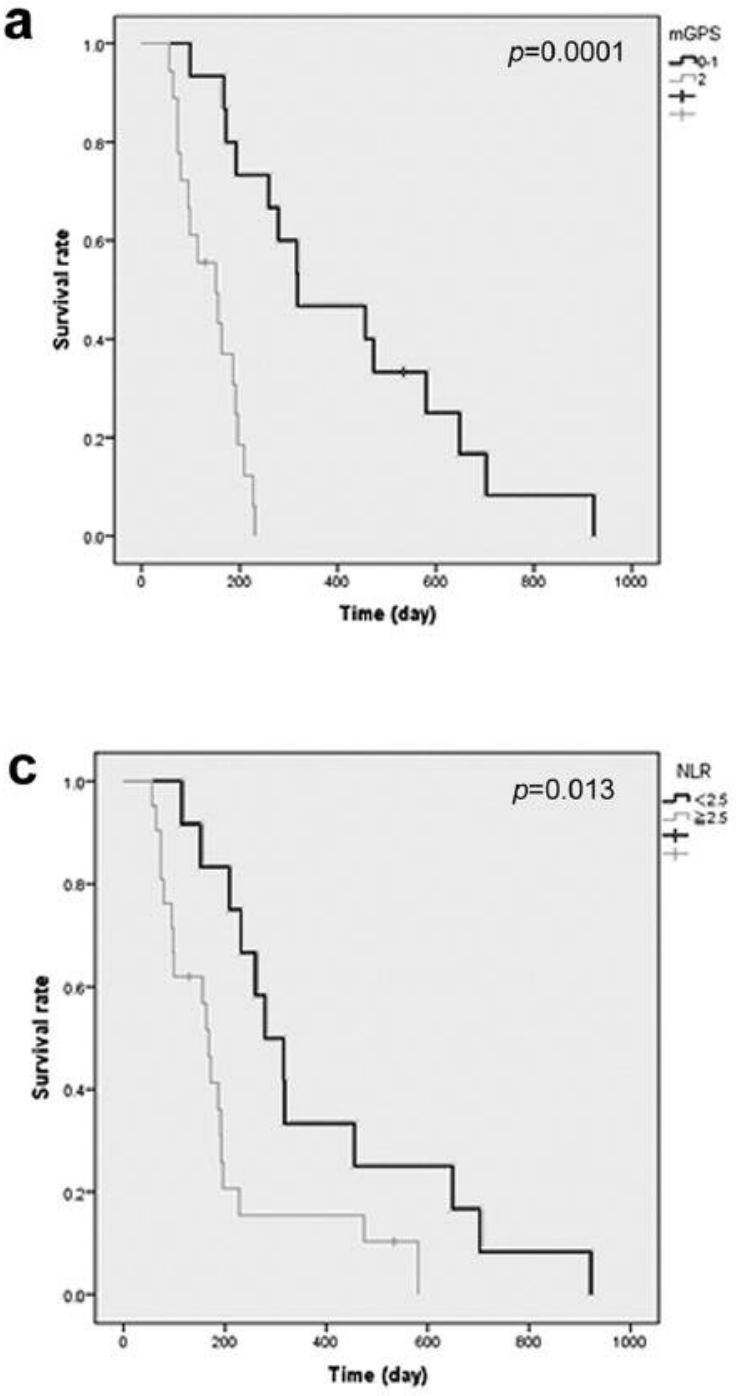
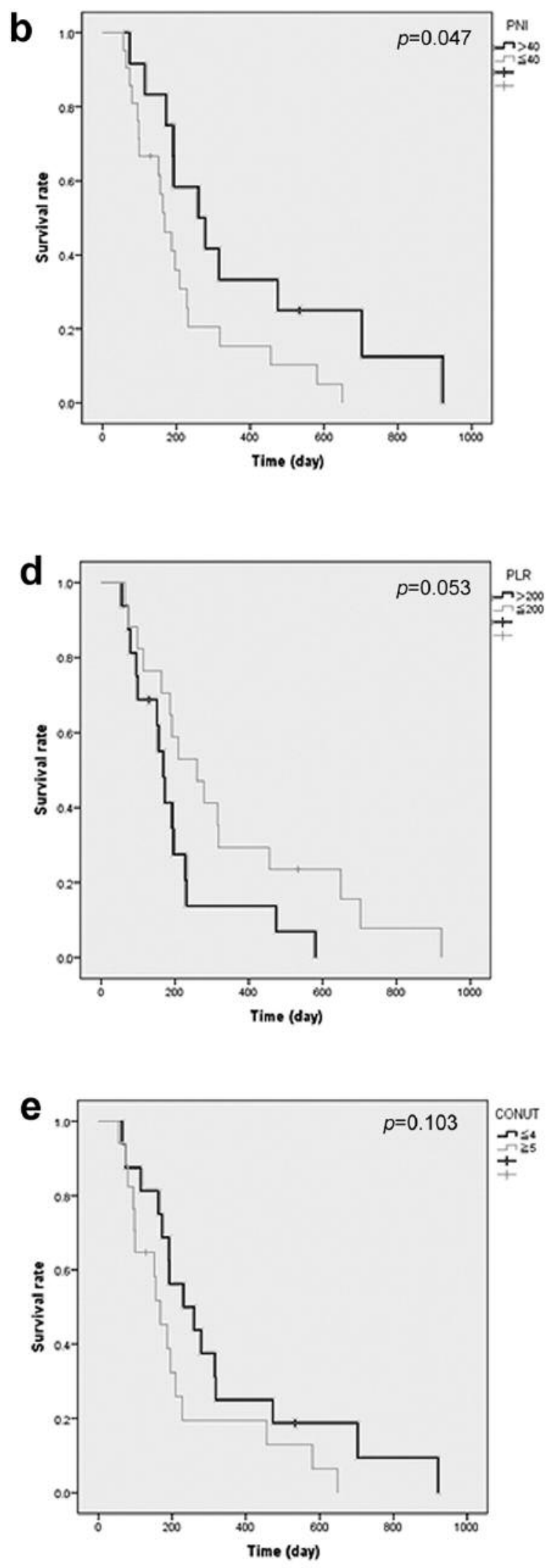

Figure 1. Relationship between prognostic indicators and overall survival in patients. a) mGPS, b) PNI, c) NLR, d) PLR, e) CONUT. 
Table II. Univariate and multivariate analyses of overall survival in patients.

\begin{tabular}{|c|c|c|c|c|c|c|c|}
\hline \multirow[t]{2}{*}{ Clinicopathological factor } & \multirow[t]{2}{*}{ Category } & \multicolumn{3}{|c|}{ Univariate analysis } & \multicolumn{3}{|c|}{ Multivariate analysis } \\
\hline & & HR & $95 \% \mathrm{CI}$ & $p$-Value & HR & $95 \% \mathrm{CI}$ & $p$-Value \\
\hline Age & $<70$ & 1.083 & $0.755-1.555$ & 0.8030 & & & \\
\hline Gender & Female & 0.604 & $0.208-1.753$ & 0.3540 & & & \\
\hline BMI & $<22$ & 0.906 & $0.623-1.319$ & 0.6080 & & & \\
\hline PS & $0-1$ & 0.909 & $0.533-1.549$ & 0.7250 & & & \\
\hline ASA & $2-4$ & 1.011 & $0.703-1.453$ & 0.9540 & & & \\
\hline Histology & PD, UD & 1.006 & $0.698-1.452$ & 0.8240 & & & \\
\hline Tumor depth & $\leq \mathrm{T} 3$ & 1.051 & $0.692-1.596$ & 0.8650 & & & \\
\hline Liver metastasis & Negative & 0.800 & $0.536-1.192$ & 0.1790 & & & \\
\hline Lung metastasis & Negative & 0.605 & $0.287-1.276$ & 0.1930 & & & \\
\hline Peritoneal metastasis & Negative & 0.735 & $0.464-1.165$ & 0.1900 & & & \\
\hline Peritoneal cytology & Negative & 1.221 & $0.843-1.767$ & 0.2040 & & & \\
\hline Number of metastatic sites & $\geq 2$ & 1.354 & $0.926-1.979$ & 0.0760 & & & \\
\hline CEA & $<5$ & 0.887 & $0.618-1.273$ & 0.5160 & & & \\
\hline CA19-9 & $\leq 37$ & 0.922 & $0.643-1.321$ & 0.6570 & & & \\
\hline Surgical treatment & Gastrojejunostomy & 1.469 & $0.921-2.345$ & 0.1070 & & & \\
\hline Postoperative complication & Absent & 0.894 & $0.565-1.408$ & 0.4440 & & & \\
\hline Chemotherapy & Not performed & 2.244 & $1.401-3.594$ & 0.0010 & 1.409 & $0.577-1.908$ & 0.8740 \\
\hline mGPS & $0-1$ & 0.336 & $0.191-0.593$ & 0.0001 & 0.371 & $0.181-0.760$ & 0.0070 \\
\hline PNI & $>40$ & 0.674 & $0.453-1.005$ & 0.0530 & & & \\
\hline NLR & $<2.5$ & 0.344 & 0.149-0.790 & 0.0120 & 0.700 & $0.452-1.083$ & 0.1090 \\
\hline PLR & $>200$ & 1.444 & $0.988-2.111$ & 0.0580 & & & \\
\hline CONUT & $\leq 4$ & 0.738 & $0.509-1.069$ & 0.0610 & & & \\
\hline
\end{tabular}

stage IV gastric cancer. In the present study, although the OS rate of PNI $>40$ group was significantly better than the PNI $\leq 40$ group, the PNI was not an independent prognostic indicator after non-curative surgery in patients with stage IV gastric cancer from a result of multivariable analysis.

It is generally known that lymphopenia is a surrogate of impaired cell-mediated immunity, whereas neutrophilia is a response to systemic inflammation. The NLR, which is calculated as the neutrophilia count divided by the lymphocyte count, has been suggested as a marker of the immune response to systemic inflammation in patients with various malignancies. It is thought that a high NLR reflects increased systemic inflammation in the host and that it is associated with a poor prognostic outcome. A meta-analysis to investigate the association between the NLR and the prognosis of gastric cancer revealed that a high preoperative NLR is associated with poor survival in patients with gastric cancer (16). Several studies have demonstrated that the NLR is a prognostic and predictive biomarker in patients with advanced gastric cancer (17-21). Yamanaka et al. (17) revealed that the NLR is an independent prognostic factor in advanced gastric cancer patients undergoing palliative chemotherapy. Jung et al. (18) revealed that an elevated pre-operative NLR predicted poor OS following resection in patients with stage III-IV gastric cancer. Jin et al. (19) revealed that the NLR before chemotherapy and surgery were independent prognostic factors for progressionfree survival in patients with stage III-IV gastric cancer undergoing neoadjuvant chemotherapy. However, to the best of our knowledge, the prognostic significance of the NLR in stage IV gastric cancer patients undergoing palliative surgery has rarely been studied. In the present study, although the NLR was a useful prognostic marker, it was not an independent prognostic factor for such patients.

The PLR is a prognostic marker in several types of malignancies (24). The relationship between the PLR and a poor prognosis may be explained through inflammatory processes caused by cancer cells. Systemic inflammation results in the release of various immunological mediators such as interleukin (IL)-1 and IL-6, which accelerate megakaryocyte proliferation leading to thrombocytosis (36). Thrombocytosis is considered to be a negative prognostic marker in gastric cancer (37). Meanwhile, lymphocytes play an important role in immune-surveillance for cancer, and prevent tumor growth (38). Lymphocytopenia has been reported to be associated with a poorer prognosis in patients with gastrointestinal malignancies, including gastric cancer (39). Thus, thrombocytosis and lymphocytopenia are both correlated with the degree of systemic inflammation in the host and are associated with the prognosis of tumor growth. However, in the present study, the PLR was not a useful 
prognostic marker in stage IV gastric cancer patients who underwent non-curative surgery.

The CONUT, which is calculated by the serum albumin concentration, the total peripheral lymphocyte count, and the total cholesterol concentration, was developed as a screening tool to allow the early detection of a poor nutritional status (31). In contrast to the GPS, PNI, NLR and PLR, the total cholesterol concentration is included in the calculation of the CONUT. Cholesterol is an essential component of the cell membrane that is involved in many of the biochemical pathways that are potentially correlated with the initiation and progression of cancer and the immune response (26). Several studies have suggested that a low serum total cholesterol level is associated with mortality in gastrointestinal duct cancer $(40,41)$. To date, a few studies have reported the CONUT as an independent prognostic factor in colorectal cancer (25) and esophageal cancer (26). However, there have been no reports on the relationship between the CONUT and the prognostic outcome in gastric cancer. The present study is the first report to evaluate the relationship between the CONUT and the survival of patients with stage IV gastric cancer. Unfortunately, there was no significant difference in the survival of stage IV gastric cancer patients with a CONUT of $<4$ and those with a CONUT of $\geq 5$.

The present study is associated with several limitations. First, it was a retrospective study that was conducted at a single institution and included a small number of patients. Furthermore, a number of patients had to be excluded due to insufficient data. Thus, well-designed prospective studies that include a large number of advanced gastric cancer patients are required. Moreover, further investigations are necessary to determine whether the cut-off mGPS, PNI, NLR, PLR and CONUT value were correct.

In conclusion, the mGPS and NLR were useful prognostic factors that predicted postoperative survival in patients undergoing non-curative surgical treatment for stage IV gastric cancer. Moreover, the preoperative mGPS was the most useful prognostic factor for such patients.

\section{Conflicts of Interest}

The Authors have no conflicts of interest to declare.

\section{References}

1 Sadi RF, Remine SG, Dudrick PS and Hanna NN: Is there a role for palliative gastrectomy in patients with stage IV gastric cancer? World J Surg 30: 21-27, 2006.

2 Roxburgh CS, and McMillan DC: Role of systemic inflammatory response in predicting survival in patients with primary operable cancer. Future Oncol 6: 149-163, 2010.

3 Schwegler I, von Holzen A, Gutzwiller JP, Schlumpf R, Mühlebach S and Stanga Z: Nutritional risk is a clinical predictor of postoperative mortality and morbidity in surgery for colorectal cancer. Br J Surg 97: 92-97, 2010.
4 Liu X, Qiu H, Liu J, Chen S, Xu D, Li W, Zhan Y, Li Y, Chen Y, Zhou Z and Sun X: A novel prognostic score, based on preoperative nutritional status, predicts outcomes of patients after curative resection for gastric cancer. J Cancer 7: 21482156, 2016.

5 Watanabe J, Otani S, Sakamoto T, Arai Y, Hanaki T, Amishima M, Tokuyasu N, Honjo S and Ikeguchi M: Prognostic indicators based on inflammatory and nutritional factors after pancreaticoduodenectomy for pancreatic cancer. Surg Today 46: 1258-1267, 2016.

6 Liu X, Sun X, Liu J, Kong P, Chen S, Zhan Y and Xu D: Preoperative C-reactive protein/albumin ratio predicts prognosis of patients after curative resection for gastric cancer. Transl Oncol 8: 339-345, 2015.

7 Kishiki $\mathrm{T}$, Masaki $\mathrm{T}$, Matsuda $\mathrm{H}$, Abe $\mathrm{N}$, Mori $\mathrm{T}$ and Sugiyama T: New prognostic scoring system for incurable stage IV colorectal cancer. Asian Pac J Cancer Prev 17: $597-$ 601, 2016.

8 Nozoe T, Iguchi T, Egashira A, Adachi E, Matsukuma A and Ezaki T: Significance of modified Glasgow prognostic score as a useful indicator for prognosis of patients with gastric carcinoma. Am J Surg 201: 186-191, 2011.

9 Kubota T, Hiki N, Nunobe S, Kumagai K, Aikou S, Watanabe $\mathrm{R}$, Sano T and Yamaguchi T: Significance of the inflammation based Glasgow prognostic score for short- and long-term outcomes after curative resection of gastric cancer. J Gastrointest Surg 16: 2037-2044, 2012.

10 Jeong JH, Lim SM, Yun JY, Rhee GW, Lim JY, Cho JY and Kim YR: Comparison of two inflammation-based prognostic scores in patients with unresectable advanced gastric cancer. Oncology 83: 292-299, 2012.

11 Wang DS, Ren C, Qiu MZ, Luo HY, Wang ZQ, Zhang DS, Wang $\mathrm{FH}, \mathrm{Li} \mathrm{YH}$ and $\mathrm{Xu} \mathrm{RH}$ : Comparison of the prognostic value of various preoperative inflammation-based factors in patients with stage III gastric cancer. Tumour Biol 33: 749-756, 2012.

12 Mimatsu k, Oida T, Fukino N, Kano H, Kawasaki A, Kida K, Kuboi Y and Amano S: Glasgow prognositic score is a useful predictive factor of outcome after palliative gastrectomy for stage IV gastric cancer. Anticancer Res 34: 3131-3136, 2014.

13 Migita K, Takayama T, Saeki K, Matsumoto S, Wakatsuki K, Enomoto K, Tanaka T, Ito M, Kurumatani N and Nakajima Y: The prognostic nutritional index predicts long-term outcomes of gastric cancer patients independent of tumor stage. Ann Surg Oncol 20: 2647-2654, 2013.

14 Sun K, Chen S, Xu J, Li G and $\mathrm{He} \mathrm{Y:} \mathrm{The} \mathrm{prognostic}$ significance of the prognostic nutritional index in cancer: a systematic review and meta-analysis. J Cancer Res Clin Oncol 140: 1537-1549, 2014.

15 Yang Y, Gao P, song Y, sun J, Chen X, Zhao J, Ma B and Wang $\mathrm{Z}$ : The prognostic nutritional index is a predictive indicators of prognosis and postoperative complications in gastric cancer: A meta-analysis. Eur J Surg Oncol 42: 1176-1182, 2016.

16 Chen J, Hong D, Zhai Y and Shen P: Meta-analysis of association between neutrophil-to-lymphocyte ratio and prognosis of gastric cancer. W J Surg Oncol 13: 122, 2015.

17 Yamanaka T, Matsumoto S, Teramukai S, Ishiwata R, Nagai Y and Fukushima M: The baseline ratio of neutrophils to lymphocytes is associated with patient prognosis in advanced gastric cancer. Oncology 73: 215-220, 2007. 
18 Jung MR, Park YK, Jeong O, Seon JW, Ryu SY, Kim DY and Kim YJ: Elevated preoperative neutrophil to lymphocyte ratio predicts poor survival following resection in late stage gastric cancer. J Surg Oncol 104: 504-510, 2011.

19 Jin H, Zhang G, liu X, Liu X, Chen C, Yu H, Huang X and Zhang Q: Blood neutrophil-lymphocyte ratio predicts survival for stages III-IV gastric cancer treated with neoadjuvant chemotherapy. W J Surg Oncol 11: 112, 2013.

20 Liu H, Song M, Fang F, Gao X, Zhang Z and Wang S: Prediction of chemotherapeutic efficacy using the ratio of neutrophils to lymphocytes in patients with unresectable or recurrent gastric cancer. Oncology Letters 10: 2244-2248, 2015.

21 Aldemir MN, Turkeli M, Simsek M, Yildirim N, Bilen Y, Yetimoglu H, Bilici $M$ and Tekin SB: Prognostic value of baseline neutrophil-lymphocyte and platelet-lymphocyte ratios in local and advanced gastric cancer patients. Asian Pac J Cancer Prev 16: 5933-5937, 2015.

22 Aliustaoglu M, Bilici A, Ustaalioglu BB, Konya V, Gucun M, Seker M and Gumus M: The effect of peripheral blood values on prognosis of patients with locally advanced gastric cancer before treatment. Med Oncol 27: 1060-1065, 2010.

23 Kitano Y, Yamashita Y, Yamaura K, Arima K, Kaida T, Miyata T, Nakagawa S, Mima K, Imai K, Hashimoto D, Chikamoto A and Baba $\mathrm{H}$ : Effects of preoperative neutrophil-to-lymphocyte and platelet -to-lymphocyte ratios on survival in patients with extrahepatic cholangiocarcinoma. Anticancer Res 37: 3229-3237, 2017.

24 Zhou X, Du Y, Huang Z, Xu J, Qin T, Wang J, Wang T, Zhu W and Liu P: Prognostic value of PLR in various cancers: A metaanalysis. PLoS One 9(6): e101119, 2014.

25 Iseki Y, Shibutani M, Maeda K, Nagahara H, Ohtani H, Sugano K, Ikeya T, Muguruma K, Tanaka H, Toyokawa T, Sakurai and Hirakawa K: Impact of the preoperative controlling nutritional status (CONUT) score on the survival after curative surgery for colorectal cancer. PLoS One 10(7): e0132488, 2015.

26 Toyokawa T, Kubo N, Tamura T, Sakurai K, Amano R, Tanaka H, Muguruma K, Yashiro M, Hirakawa K and Ohira M: The pretreatment controlling nutritional status (CONUT) score is an independent prognostic factor in patients with resectable thoracic esophageal squamous cell carcinoma: results from a retrospective study. BMC Cancer 16: 722, 2016.

27 Shoji F, Haratake N, Akamie T, Takamori S, Katsura M, Takada $\mathrm{K}$, Toyokawa G, Okamoto $\mathrm{T}$ and Maehara Y: The preoperative controlling nutritional status score predicts survival after curative surgery in patients with pathological stage I non-small cell lung cancer. Anticancer Res 37: 741-747, 2017.

28 Sobin LH, Gospodarowicz MK and Wittekind $\mathrm{CH}$ : International Union against Cancer (UICC) TNM classification of malignant tumors, 7th edn. Wiley-Blackwell, Oxford, 2009.

29 Toiyama Y, Miki C, Inoue Y, Tanaka K, Mohri Y and Kusunoki $\mathrm{M}$ : Evaluation of an inflammation-based prognostic score for the chemotherapy for stage II colorectal cancer. Exp Ther Med 2: 95-101, 2011.
30 Onodera T, Goseki N and Kosaki G: Prognostic nutritional index in gastrointestinal surgery of malnurished cancer patients. Nihon Geka Gakkai Zasshi 85: 1001-1005, 1984.

31 Ulibarri JI, Gonzalez-Madrono A, De Villar NGP, Gonzalez P, Gonzalez B, Mancha A, Rodriguez F and Fernandez G: CONUT: A tool for controlling nutritional status. First validation in a hospital population. Nutr Hosp 20: 38-45, 2005.

32 Dindo D, Demartines $\mathrm{N}$ and Clavien PA: Classification of surgical complications: a new proposal with evaluation in a cohort of 6336 patients and results of a survey. Ann Surg 240: 205-213, 2004.

33 McMillan DC, Elahi MM, Sattar N, Anderson WJ, Johnstone J and McArdle CS: Measurement of systematic inflammatory response predicts cancer-specific and non-cancer survival in patients with cancer. Nutr Cancer 41: 64-69, 2001.

34 Nozoe T, Iguchi T, Adachi E, Matsukuma A and Ezaki T: Preoperative elevation of serum $\mathrm{C}$-reactive protein as an independent prognostic indicator for gastric cancer. Surg Today 41: 510-513, 2011.

35 Buzby GP, Mullen JL, Matthews DC, Hobbs CL and Rosato EF: Prognostic nutrition index in gastrointestinal surgery. Am J Surg 139: 160-167, 1980.

36 Alexandrakis MG, Passam FH, Moschandrea IA, Christophoridou AV, Pappa CA, Coulocheri SA and Kyriakou DS: Levels of serum cytokines and acute phase proteins in patients with essential and cancer-related thrombocytosis. Am J Clin Oncol 26: 135-140, 2003.

37 Ikeda M, Furukawa H, Imamura H, Shimizu J, Ishida H, Masutani S, Tatsuta M and Satomi T: Poor prognosis associated with thrombocytosis in patients with gastric cancer. Ann Surg Oncol 9: 287-291, 2002.

38 Dunn GP, Old LJ and Schreiber RD: The immunobiology of cancer innumosurveillance and immunoediting. Immunity 21 : 137-148, 2004.

39 Romano F, Uggeri F, Crippa S, Di Stefano G, Scotti M, Scaini A, Caprotti R and Uggeri F: Immunodeficiency in different histotypes of radically operable gastrointestinal cancers. J Exp Clin Cancer Res 23: 195-200, 2004.

40 Sanz L, Ovejero VJ, Gonzalez JJ, Laso CA, Azcano E, Navarrete $\mathrm{F}$ and Martínez E: Mortality risk scales in esophagectomy for cancer: their usefulness in preoperative patient selection. Hepatogastroenterology 53: 869-873, 2006.

41 Cengiz O, Kocer B, Sumeli S, Stanicky MJ and Soran A: Are pretreatment serum albumin and cholesterol levels prognostic tools in patients with colorectal carcinoma? Med Sci Monit 12: CR240-247, 2006. 